\title{
Epigenetic effects of the natural flavonolignan silibinin on colon adenocarcinoma cells and their derived metastatic cells
}

\author{
HENRIETTE KAUNTZ ${ }^{1,2}$, SOUAD BOUSSEROUEL ${ }^{1,2}$, FRANCINE GOSSÉ ${ }^{1,2}$ and FRANCIS RAUL FR $^{1,2}$ \\ ${ }^{1}$ Department of Nutritional Cancer Prevention, University of Strasbourg, Unit EA 4438, Faculty of Medicine; \\ ${ }^{2}$ IRCAD-EITS, F-67000 Strasbourg, France
}

Received October 25, 2012; Accepted January 18, 2013

DOI: $10.3892 / \mathrm{ol} .2013 .1190$

\begin{abstract}
Epigenetic modifications are important in tumorigenesis. The most frequent epigenetic phenomena in cancer are histone deacetylation and DNA hypermethylation, which lead to gene silencing, particularly of tumor suppressor genes. However, monotherapies with histone deacetylase (HDAC) or DNA methyltransferase (DNMT) inhibitors lack efficacy, hence there is a need to enhance their anticancer action in a safe and effective combination therapy. The present study investigated the epigenetic effects of the natural flavonolignan silibinin in a model of colon cancer progression, the primary adenocarcinoma cells SW480 and their derived metastatic cells SW620. Silibinin did not change the activity of HDACs, but it was able to significantly inhibit DNMT activity in both SW480 and SW620 cells. The clinically used HDAC inhibitor, suberoylanilide hydroxamic acid (SAHA), and the broad spectrum HDAC inhibitor, trichostatin A (TSA), combined with silibinin demonstrated synergistic effects on cell death induction, may be related to its DNMT inhibition properties. The present data suggest that treatments combining silibinin and HDAC inhibitors may represent a promising approach, given the non-toxic nature of silibinin and the fact that HDAC inhibitors selectively target cancer cells.
\end{abstract}

\section{Introduction}

Epigenetic modifications affect gene expression. Tumors often show an aberrant epigenetic modification pattern, including histone deacetylation and DNA hypermethylation, which leads to the suppression of gene expression (1). Colorectal cancer (CRC) in particular results not only from an accumulation of genetic changes, but also epigenetic changes, which occur in the course of the transformation of normal epithelium into adenocarcinomas.

Correspondence to: Dr Francis Raul, IRCAD, 1 Place de l'Hôpital, F-67091 Strasbourg-Cedex, France

E-mail: francis.raul@ircad.u-strasbg.fr

Key words: colorectal cancer, silibinin, HDAC inhibitors, DNMT inhibitors, combination therapy
Histone deacetylases (HDACs) catalyze the removal of acetyl groups, thereby stimulating chromatin condensation, which promotes transcriptional repression, including the shutdown of tumor suppressor gene expression (2). HDACs are also able to target numerous non-histone proteins, such as p53, even accounting for a majority of HDAC substrates (3). Overexpression of HDACs and epigenetic silencing has been frequently observed in CRC (4,5). Amongst others, these studies show that the perturbation of the balance between acetylation and deacetylation plays an important role in neoplastic transformation. As epigenetic changes are reversible, HDACs constitute promising targets for pharmacological inhibition in CRC (3).

By mechanisms not yet fully elucidated, HDAC inhibitors are able to induce cell-cycle arrest, promote differentiation and selectively stimulate apoptosis in transformed cells via the extrinsic and/or intrinsic apoptotic pathway (6). Furthermore, they synergistically enhance the anticancer activity of chemotherapeutic drugs, particularly those that are pro-apoptotic, by shifting the balance between pro- and anti-apoptotic proteins.

Examples of HDAC inhibitors include short-chain fatty acids, hydroxamic acids, benzamides and cyclic tetrapeptides (2). The broad spectrum HDAC inhibitor SAHA (also known as Zolinza ${ }^{\circledR}$ or Vorinostat) was the first HDAC inhibitor to have successfully completed clinical trials and is used for the treatment of cutaneous T-cell lymphoma (7). Numerous HDAC inhibitors are currently undergoing clinical trials (2). TSA is another hydroxamic acid and broad spectrum HDAC inhibitor which targets class I, II and IV HDACs in the same way as SAHA (3).

DNA hypermethylation is another frequent phenomenon in cancer that silences many genes for cell cycle regulation, receptors and apoptosis by DNA methylation of $\mathrm{CpG}$ islands in their promoter region (8). A proportion of proximal colon tumors (30-40\%), and distal and rectal tumors (3-12\%), exhibit a $\mathrm{CpG}$ island methylator phenotype, where numerous $\mathrm{CpG}$ islands are methylated and various tumor suppressor genes are inactivated (9).

DNA methyltransferase (DNMT) inhibitors are able to induce DNA demethylation and thus the reactivation of epigenetically silenced genes. At present, there are two FDA-approved drugs with DNMT inhibitory activity: 5-azacytidine and decitabine, both of which are used in the 
treatment of myelodysplastic syndrome and myeloid leukemias (3). Global reduction of DNA methylation has been demonstrated to have anticancer effects in intestinal tumorigenesis (10).

Histone deacetylation has been revealed to act synergistically with DNA methylation in the epigenetic silencing of cancer genes (11). Therefore combinations of DNMT and HDAC inhibitors may have potential for further clinical trials (10).

Natural substances have been found to act on epigenetic signaling; green tea polyphenols demonstrated similar effects to TSA in prostate cancer cells by inducing cell cycle arrest and apoptosis (12). These polyphenols were able to inhibit HDACs and induce their proteasomal degradation. Furthermore, the tea polyphenol (-)-epigallocatechin-3-gallate was also able to inhibit DNMT activity and reactivate methylation-silenced genes in CRC cells (13). In addition, apple polyphenols reduced DNA methylation by inhibition of DNMT in CRC cells (14). Moreover, soy isoflavones reversed DNA hypermethylation and reactivated silenced genes in esophageal squamous cell carcinoma cells (15), and their anticancer activity was enhanced when used in combination with HDAC inhibitors (15). The polyphenol curcumin has also been shown to inhibit DNMT activity (16) and to act synergistically with TSA in inducing cell death (17).

The flavonolignan silibinin, which is the main pharmacologically active component of the milk thistle plant (Silybum marianum), has been shown to increase acetylation of histones in hepatic cancer in vitro and in vivo. Silibinin increased acetylation of histone $\mathrm{H} 3$ and $\mathrm{H} 4$ in vitro in $\mathrm{HuH} 7$ cells (18), and in vivo in HuH7 xenografts in nude mice (19). In non-small cell lung cancer, silibinin inhibited HDAC activity and decreased HDAC levels (20).

However, no studies have yet to fully describe the effect of silibinin on DNMT activity. Previously, we demonstrated that silibinin exerted anti-proliferative and pro-apoptotic effects in the primary adenocarcinoma SW480 cells and in their metastatic derivatives (SW620 cells) (21). In the present study, we aimed to investigate whether silibinin modified HDAC and DNMT activity in this model of CRC progression.

\section{Material and methods}

Cell culture and treatment. SW480 and SW620 cells were purchased from the European Collection of Animal Cell Culture (ECACC, Salisbury, UK). The cells were cultured in $75 \mathrm{~cm}^{2}$ Falcon flasks in Dulbecco's modified Eagle's medium (DMEM) containing $25 \mathrm{mM}$ glucose and supplemented with $10 \%$ heat-inactivated $\left(56^{\circ} \mathrm{C}\right)$ horse serum, $100 \mathrm{U} / \mathrm{ml}$ penicillin, $100 \mu \mathrm{g} / \mathrm{ml}$ streptomycin and $1 \%$ non-essential amino acids (Invitrogen Life Technologies, Lyon, France). Cells were maintained at $37^{\circ} \mathrm{C}$ in a humidified atmosphere with $5 \% \mathrm{CO}_{2}$, and subcultured following trypsinization ( $0.5 \%$ trypsin $/ 2.6 \mathrm{mM}$ ethylendiamine tetraacetic acid). For all experiments, cells were seeded at $1 \times 10^{6}$ cells in culture dishes (10 cm internal diameter). The culture medium used was DMEM supplemented with 3\% heat-inactivated horse serum and $100 \mathrm{U} / \mathrm{ml}$ penicillin, $100 \mu \mathrm{g} / \mathrm{ml}$ streptomycin and $1 \%$ non-essential amino acids. Additionally, $5 \mu \mathrm{g} / \mathrm{ml}$ transferrin, $5 \mathrm{ng} / \mathrm{ml}$ selenium and $10 \mu \mathrm{g} / \mathrm{ml}$ insulin were added to compensate for the lower serum concentration. The culture medium was replaced every $48 \mathrm{~h}$. Cells were exposed to silibinin (Sigma-Aldrich Chemie GmbH, Steinheim, Germany) $24 \mathrm{~h}$ after seeding. Silibinin was dissolved in dimethylsulfoxide (DMSO; Sigma-Aldrich Chemie $\mathrm{GmbH}$ ) and used at a final concentration of $300 \mu \mathrm{M}$. The final concentration of DMSO in the culture medium did not exceed $0.1 \%$. The HDAC inhibitors, TSA $(0.1 \mu \mathrm{M})$ or SAHA $(1 \mu \mathrm{M})$ (SigmaAldrich Chemie $\mathrm{GmbH}$ ), were added $1 \mathrm{~h}$ prior to silibinin treatment.

Histone deacetylase (HDAC) and DNA methyltransferase (DNMT) activity. To determine the activity/inhibition of the HDACs/DNMTs in the nuclear samples, the colorimetric EpiQuik ${ }^{\mathrm{TM}}$ HDAC Activity/ Inhibition Assay kit (Epigentek-Euromedex, Strasbourg, France) and the colorimetric EpiQuik ${ }^{\mathrm{TM}}$ DNMT Activity/ Inhibition Assay Ultra kit (Epigentek-Euromedex) were used. Cells were harvested by scraping, and nuclear extracts of the cells were prepared using the EpiQuik ${ }^{\mathrm{TM}}$ Nuclear Extraction kit (Epigentek-Euromedex). The protein content of the nuclear extracts was determined by the Lowry assay, and any nuclear extracts not immediately used were stored at $-80^{\circ} \mathrm{C}$.

To measure the HDAC activity, the same quantity of nuclear extract was incubated with acetylated histone substrate in a 96-well plate, and the quantity of remaining un-deacetylated histone that is inversely proportional to HDAC enzyme activity in the nuclear sample was colorimetrically quantified through an ELISA-like reaction at a wavelength of $450 \mathrm{~nm}$. The activity of HDAC enzymes was inversely proportional to the OD.

To measure the DNMT activity, nuclear extracts were incubated on a microplate stably coated with a universal DNMT substrate. DNMT enzymes from the nuclear sample methylate the DNA substrate during incubation, and then the quantity of methylated DNA that is proportional to enzyme activity was colorimetrically quantified through an ELISA-like reaction at a wavelength of $450 \mathrm{~nm}$. The activity of DNMT enzymes was proportional to the OD.

Flow cytometric analysis of the sub-G0/G1 cell population. The sub-G0/G1 cell population (hypodiploid cells: dying and dead cells) was analyzed by labeling cells with propidium iodide. Cells were harvested by trypsinization after 24, 48 and $72 \mathrm{~h}$ of treatment and washed with phosphate-buffered saline (PBS; $0.1 \mathrm{M}$; pH 7.4). Cells were fixed in $70 \%$ ethanol at $-20^{\circ} \mathrm{C}$ for $\geq 30 \mathrm{~min}$, washed twice with PBS and re-suspended in $200 \mu \mathrm{l}$ PBS containing $0.25 \mathrm{mg} / \mathrm{ml}$ RNase A and $0.1 \mathrm{mg} / \mathrm{ml}$ propidium iodide (Sigma-Aldrich Chemie $\mathrm{GmbH}$ ). Following incubation in the dark at $37^{\circ} \mathrm{C}$ for $30 \mathrm{~min}$, the fluorescence of 10,000 cells/sample was analyzed by flow cytometry, and histograms were analyzed using the CellQuest software (FACScan, BD Biosciences, Belgium).

Statistical analysis. All experiments were performed $\geq 3$ times. Data are presented as mean \pm standard error. Statistical differences between the control and treated groups were evaluated using the Student's t-test or the Student-Newman-Keuls multiple comparison test. $\mathrm{P}<0.05$ was considered to indicate a statistically significant difference between groups. 
A

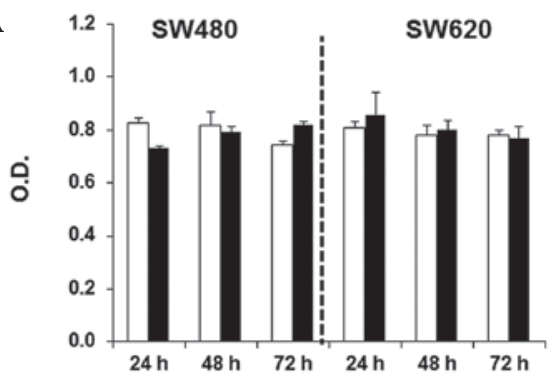

B

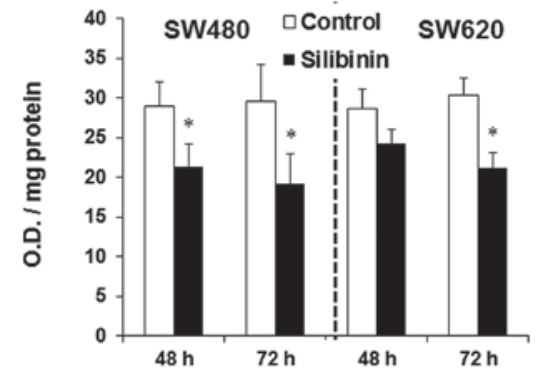

Figure 1. Effect of silibinin on HDAC and DNMT activity. SW480 and SW620 cells were treated with DMSO $0.1 \% \pm$ silibinin $(300 \mu \mathrm{M})$ for 24 , 48 or $72 \mathrm{~h}$. Nuclear extracts were prepared, and HDAC (A) or DNMT (B) activity was measured by colorimetric methods as detailed in Materials and methods. Data are presented as the mean \pm standard error of three separate experiments. Data are expressed as the OD for equal amounts of protein (A) or as $\mathrm{OD} / \mathrm{mg}$ protein (B). For each cell line, silibinin treatment vs. non-treated control: ${ }^{\mathrm{P}} \mathrm{P}<0.05$
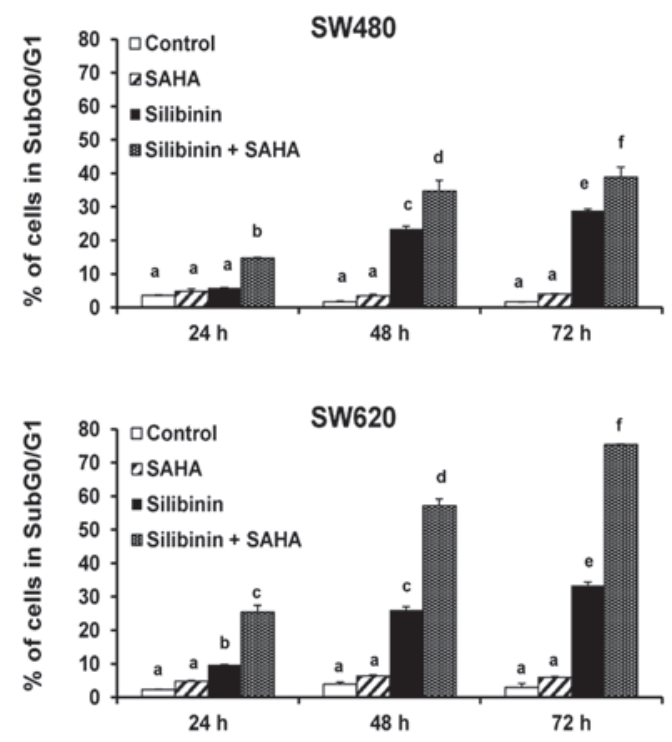

Figure 2. Cell death induced by silibinin and SAHA. SW480 and SW620 cells were treated with DMSO $0.1 \% \pm$ silibinin $(300 \mu \mathrm{M}) \pm$ SAHA $(1 \mu \mathrm{M})$ for 24,48 or $72 \mathrm{~h}$. At each time point, SW480 and SW620 cells were harvested and stained with propidium iodide for the measurement of hypodiploid bodies and analyzed by flow cytometry as detailed in Materials and methods. Data are presented as the mean \pm standard error of three separate experiments. For each cell line, columns that do not share the same superscript differ significantly: " $\mathrm{P}<0.05$

\section{Results}

Effects of silibinin on HDAC and DNMT activity. To establish whether silibinin induced epigenetic modifications in SW480 and SW620 cells, we determined HDAC and DNMT
A

SW480

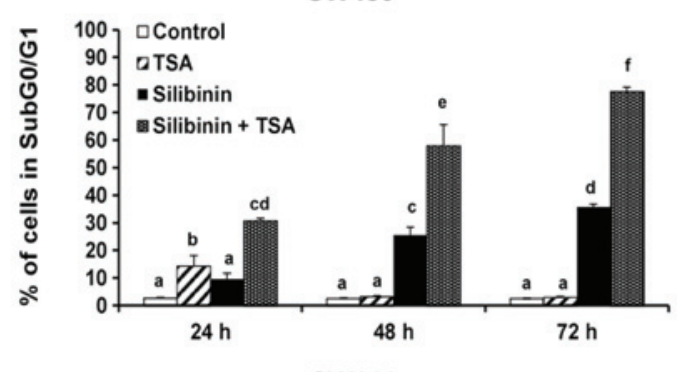

SW620

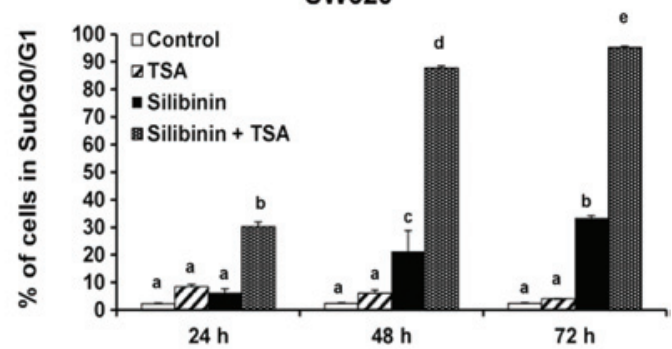

B

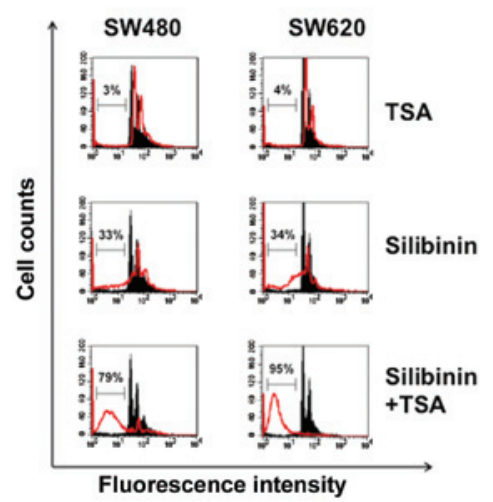

Figure 3. Cell death induced by silibinin and TSA. SW480 and SW620 cells were treated with DMSO $0.1 \% \pm$ silibinin $(300 \mu \mathrm{M}) \pm$ TSA $(0.1 \mu \mathrm{M})$ for 24 , 48 or $72 \mathrm{~h}$. At each time point, SW480 and SW620 cells were harvested and stained with propidium iodide for the measurement of hypodiploid bodies and analyzed by flow cytometry as detailed in Materials and methods. In (A), data are presented as the mean \pm standard error of three separate experiments. For each cell line, columns that do not share the same superscript differ significantly: "P<0.05. (B) representative FACS histograms at $72 \mathrm{~h}$; the black histogram represents the DMSO-only control cells while the red line represents the treated cells. The percentage of cells in the subG0/G1 region is indicated.

activity in nuclear extracts of silibinin-treated cells with the aid of the colorimetric EpiQuik HDAC Activity/Inhibition Assay kit and the EpiQuik DNMT Activity/Inhibition Assay Ultra kit.

HDAC activity in SW480 and SW620 cells was not changed by silibinin treatment (Fig. 1A). In contrast, a reduction in DNMT activity was observed in silibinin-treated SW480 and SW620 cells after $48 \mathrm{~h}$ of treatment with silibinin (Fig. 1B). However, this reduction only became significant following $72 \mathrm{~h}$ of treatment in SW620 cells.

Silibinin and HDAC inhibitors induce synergistic cell death. Because synergy between DNA demethylation and HDAC inhibition has been demonstrated in the re-expression of genes silenced in cancer (11), we investigated the synergistic effect of 
silibinin, which inhibits DNMT as shown, in combination with HDAC inhibitors on cell death.

The clinically used HDAC inhibitor SAHA significantly enhanced silibinin-induced cell death (Fig. 2) but demonstrated no cell toxicity on its own. However, this effect was notably stronger in SW620 cells. To verify the interaction between silibinin and HDAC inhibitors, we used the broad spectrum HDAC inhibitor TSA and observed a clear synergistic effect with silibinin on cell death induction in both cell lines (Fig. 3). Cell death induction was more notable with TSA than with SAHA, and we found that the metastatic SW620 cells were more sensitive to the combined treatment than the adenocarcinoma SW480 cells.

\section{Discussion}

Previously, we demonstrated that the polyphenol silibinin inhibited cell growth and induced apoptosis in SW480 and SW620 cells (21). In an azoxymethane-induced rat model, silibinin was able to prevent the formation of preneoplastic lesions, thus appearing to be a promising chemopreventive agent in CRC (22). Polyphenols have been shown to modify histone deacetylation and DNA hypermethylation, accompanying their chemopreventive effect $(12-14,16,20)$.

In the present study, we investigated the epigenetic effects of silibinin. We found that silibinin did not change HDAC activity in SW480 and SW620 cells. These data contrasted with previous observations by Lah et al and Cui et al in hepatocarcinoma cells and xenografts $(18,19)$, and by Mateen et al in non-small cell lung cancer (20), where silibinin was able to inhibit the activity of HDACs. However, silibinin reduced DNMT activity in both cell lines following $72 \mathrm{~h}$ of treatment. Inhibition of DNMT activity was already significant at $48 \mathrm{~h}$ in SW480 cells.

As other polyphenols and DNMT inhibitors have been demonstrated to act synergistically with HDAC inhibitors in cell death induction, we tested the effect of a combination of silibinin with two broad-spectrum HDAC inhibitors, SAHA and TSA, on the two cell lines. Both combinations synergistically induced cell death. These results were in agreement with those of other studies demonstrating that silibinin synergistically augmented the cytotoxic effects of SAHA and TSA in non-small cell lung cancer cells (20). Notably, in our study, SW480 and SW620 cells were both resistant to treatment by the HDAC inhibitors, which alone exhibited no cytotoxic effects.

However, the synergistic effect of silibinin and HDAC inhibitors could not be entirely attributed to silibinin-induced DNMT inhibition, as the increase in cell death occurred prior to significant DNMT inhibition by silibinin in SW620 cells. Furthermore, the synergy in cell death induction was stronger in SW620 cells (up to 95\% cell death compared with $80 \%$ in SW480 cells), whereas DNMT inhibition was weaker in SW620 cells than in SW480 cells.

Silibinin and HDAC inhibitors possess pleiotropic anticancer activities, which may explain their synergistic effects; the ability of HDAC inhibitors to change the balance between pro- and anti-apoptotic factors (6) may contribute to the enhancement of the apoptosis-inducing properties of silibinin.
In conclusion, silibinin inhibited DNMT but not HDAC activity in colorectal SW480 and metastatic SW620 cells, and exerted synergistic effects with HDAC inhibitors on cancer cell death. Further investigations are required to determine the mechanisms involved in this process. However, our data suggest that treatments combining silibinin and HDAC inhibitors may represent a promising approach, given the non-toxic nature of silibinin and the fact that HDAC inhibitors selectively target cancer cells. Combined treatment of silibinin with different epigenetic agents, including HDAC inhibitors, in current clinical trials may thus contribute to the development of novel combination therapies.

\section{Acknowledgements}

Henriette Kauntz was supported by a fellowship provided by the Conseil Régional d'Alsace, France.

\section{References}

1. Lu Q, Qiu X, Hu N, Wen H, Su Y and Richardson BC: Epigenetics, disease, and therapeutic interventions. Ageing Res Rev 5: 449-467, 2006.

2. Song SH, Han SW and Bang YJ: Epigenetic-based therapies in cancer: progress to date. Drugs 71: 2391-2403, 2011.

3. Carew JS, Giles FJ and Nawrocki ST: Histone deacetylase inhibitors: mechanisms of cell death and promise in combination cancer therapy. Cancer Lett 269: 7-17, 2008.

4. Mariadason JM: HDACs and HDAC inhibitors in colon cancer. Epigenetics 3: 28-37, 2008.

5. Ashktorab H, Belgrave K, Hosseinkhah F, Brim H, Nouraie M, Takkikto M, Hewitt S, Lee EL, Dashwood RH and Smoot D: Global histone H4 acetylation and HDAC2 expression in colon adenoma and carcinoma. Dig Dis Sci 54: 2109-2117, 2009.

6. Emanuele S, Lauricella M and Tesoriere G: Histone deacetylase inhibitors: apoptotic effects and clinical implications (Review). Int J Oncol 33: 637-646, 2008.

7. Wagner JM, Hackanson B, Lübbert $M$ and Jung M: Histone deacetylase (HDAC) inhibitors in recent clinical trials for cancer therapy. Clin Epigenetics 1: 117-136, 2010.

8. Jones PA and Baylin SB: The fundamental role of epigenetic events in cancer. Nat Rev Genet 3: 415-428, 2002.

9. Migliore L, Migheli F, Spisni R and Coppedè F: Genetics, cytogenetics, and epigenetics of colorectal cancer. J Biomed Biotechnol 2011: 792362, 2011.

10. Rius M and Lyko F: Epigenetic cancer therapy: rationales, targets and drugs. Oncogene 31: 4257-4265, 2012.

11. Cameron EE, Bachman KE, Myöhänen S, Herman JG and Baylin SB: Synergy of demethylation and histone deacetylase inhibition in the re-expression of genes silenced in cancer. Nat Genet 21: 103-107, 1999.

12. Thakur VS, Gupta K and Gupta S: Green tea polyphenols causes cell cycle arrest and apoptosis in prostate cancer cells by suppressing class I histone deacetylases. Carcinogenesis 33: 377-384, 2012 .

13. Fang MZ, Wang Y, Ai N, Hou Z, Sun Y, Lu H, Welsh W and Yang CS: Tea polyphenol (-)-epigallocatechin-3-gallate inhibits DNA methyltransferase and reactivates methylation-silenced genes in cancer cell lines. Cancer Res 63: 7563-7570, 2003.

14. Fini L, Selgrad M, Fogliano V, Graziani G, Romano M, Hotchkiss E, Daoud YA, De Vol EB, Boland CR and Ricciardiello L: Annurca apple polyphenols have potent demethylating activity and can reactivate silenced tumor suppressor genes in colorectal cancer cells. J Nutr: 137: 2622-2628, 2007.

15. Fang MZ, Chen D, Sun Y, Jin Z, Christman JK and Yang CS: Reversal of hypermethylation and reactivation of p16INK4a, RARbeta, and MGMT genes by genistein and other isoflavones from soy. Clin Cancer Res 11: 7033-7041, 2005.

16. Shu L, Khor TO, Lee JH, Boyanapalli SSS, Huang Y, Wu TY, Saw CL, Cheung KL and Kong AN: Epigenetic CpG demethylation of the promoter and reactivation of the expression of Neurog1 by curcumin in prostate LNCaP cells. AAPS J 13: 606-614, 2011. 
17. Yan G, Graham K and Lanza-Jacoby S: Curcumin enhances the anticancer effects of trichostatin a in breast cancer cells. Mol Carcinog Jan 30, 2012 (Epub ahead of print).

18. Lah JJ, Cui W and Hu KQ: Effects and mechanisms of silibinin on human hepatoma cell lines. World J Gastroenterol 13: 5299-5305, 2007.

19. Cui W, Gu F and Hu KQ: Effects and mechanisms of silibinin on human hepatocellular carcinoma xenografts in nude mice. World J Gastroenterol 15: 1943-1950, 2009.

20. Mateen S, Raina K, Jain A, Agarwal C, Chan D and Agarwal R: Epigenetic modifications and p21-cyclin B1 nexus in anticancer effect of histone deacetylase inhibitors in combination with silibinin on non-small cell lung cancer cells. Epigenetics 7: 1161-1172, 2012.
21. Kauntz H, Bousserouel S, Gossé F and Raul F: Silibinin triggers apoptotic signaling pathways and autophagic survival response in human colon adenocarcinoma cells and their derived metastatic cells. Apoptosis 16: 1042-1053, 2011.

22. Kauntz H, Bousserouel S, Gossé F, Marescaux J and Raul F. Silibinin, a natural flavonoid, modulates the early expression of chemoprevention biomarkers in a preclinical model of colon carcinogenesis. Int J Oncol 41: 849-854, 2012. 